\title{
Statistical geometry of random fibrous networks, revisited: Waviness, dimensionality, and percolation
}

Y. B. Yi and L. Berhan

Department of Mechanical Engineering, University of Michigan, Ann Arbor, Michigan 48109

\author{
A. M. Sastry ${ }^{\text {a) }}$ \\ Department of Mechanical Engineering and Department of Biomedical Engineering, University \\ of Michigan, Ann Arbor, Michigan 48109
}

(Received 15 January 2004; accepted 27 April 2004)

\begin{abstract}
Waviness alters both geometric and mechanical properties of stochastic fibrous networks and significantly affects overall mechanical response, but few results are available in the literature on the subject. In this work, we explore the importance of the dimension of constituent fibers (1D vs 2D) in determination of percolation thresholds, and other fundamental statistical properties of fibers having geometric waviness, in adaptation of classical theories on random lattices to practical applications, including analysis of nanotube ropes and collagen bundles. Although the so-called "curl ratio" clearly affects the statistical properties, as evaluated by Kallmes and Corte a few decades ago, we have found some results in this classic work to be inaccurate for systems containing fibers of moderate waviness. Our main findings include the independence of the mean number of crossings with regard to waviness, as well as the nonlinear dependence of probability of intersection on waviness. Our investigation of percolation in wavy fiber networks reveals that the percolation threshold is significantly increased, with increasing curl ratio. In addition, several nontrivial results related to network properties of infinite straight lines are also described, some of which are believed to have wide applications in practice. (C) 2004 American Institute of Physics.
\end{abstract}

[DOI: $10.1063 / 1.1763240]$

\section{INTRODUCTION}

Kallmes and Corte ${ }^{1}$ considered the effects of moderate curvature in fibers on the geometry and moduli of papers several decades ago, following work on the statistical geometry of straight-fiber networks. ${ }^{2-4}$ Since that time, the behaviors of many similarly constructed systems have been studied, including "nanoarchitectured" materials, from structural proteins $^{5,6}$ to carbon nanotubes ${ }^{7,8}$ (Fig. 1). Common features of such materials include the random arrangement of fibers, their high aspect ratio (length/diameter), and their moderate to high curvature.

It is important to distinguish between fiber crossing density, a statistical quantity, and fiber bond density, which arises from the material properties and/or processing conditions for a material. However, significant fractions of fiber crossings immediately form bonds in many systems (e.g., polymer-coated paper fibers). Densities and distribution of bonds in random fibrous materials are critically related to their mechanical properties; ${ }^{9-11}$ loads are more efficiently transferred through tension (statically determinate, or triangulated systems), or a combination of tension and bending (statically indeterminate systems), than through friction (entangled systems). Comparisons of material properties, e.g., modulus, with theoretical properties of a perfectly bonded system can allow inference as to the percentage of crossings which actually form bonds. Thus, determination of the sta-

\footnotetext{
a) Author to whom correspondence should be addressed; electronic mail: amsastry@umich.edu
}

tistically predicted crossing density is an important first step in materials analysis.

Systems of infinite, straight fibers were investigated, classically, by Goudsmit ${ }^{2}$ who derived several geometric properties of such materials, including the number of polygons formed by $N$ lines, and the average number of sides per polygon. Miles ${ }^{3,4}$ later studied these systems in more detail, deriving the mean perimeter, area, and statistical distribution of number of sides of internal polygons. Richards ${ }^{12}$ reported some further statistical results, including the mean separation of two random points within the polygons, and the mean moment of inertia of the polygons. Later, Tanner ${ }^{13}$ derived the distribution of the numbers of sides of the polygons. All of these approaches relied upon analytic solutions for the probabilities calculated, for infinite systems. Direct simulations of finite networks containing fibers of finite length (i.e., having finite "staple lengths" referred to in literature on paper) have shown that they can have significantly different internal geometry than infinite fiber systems, particularly of low density. ${ }^{9,10}$

Quantitative description of random networks of wavy lines were first provided by Kallmes ${ }^{1}$ and Corte, ${ }^{1,14}$ who systematically expressed structural averages and totals in terms of the number and dimensions of the structural units. The latter quantities were termed "independent variables," including the number of lines per unit area, and the mean length and width of constituent fibers. The statistical averages and totals were termed "dependent variables," and included the total number of fiber crossings, the mean segment 


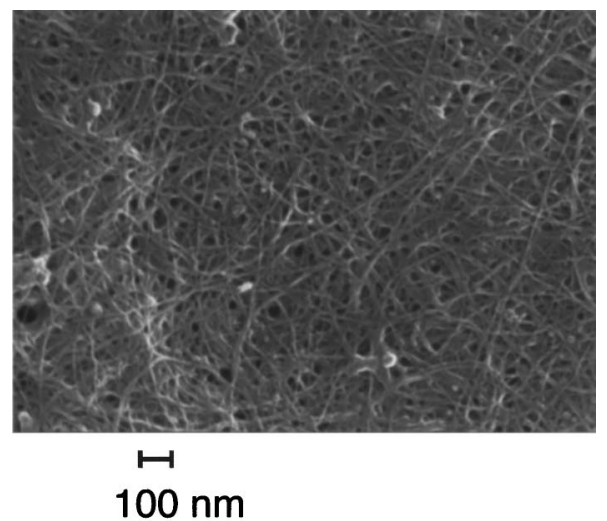

(a)

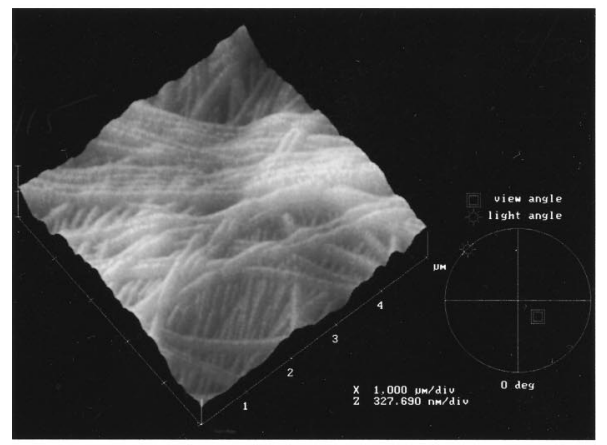

(b)

FIG. 1. (a) A scanning electron microscopy image of nanotube sheets showing the wavy fiber segments; (b) an atomic force microscopy image of epineurial collagen from a diabetic BioBreading rat sciatic nerve.

length, and total number of polygons formed. The analytical solutions derived showed the number of fiber crossings to strongly depend upon curl ratio, Kallmes' and Corte's measure of curliness. ${ }^{1}$ This rather counterintuitive finding for Poisson lines led to the present work, in which we establish several apparent errors in this classic work, and also develop some potentially useful relations for image analysis of fibrous architectures. Specifically, we investigate both analytically and numerically, in infinite and finite systems, respectively, the dependence of crossing density on curliness, mean area of intersection for finite-thickness fibers, and percolation onset. It is important to note that for two noncoincident, intersecting, straight fibers, the number of crossings is exactly the probability of intersection, because there can be one and only one crossing point. Multiple crossing points are possible, however, for two curly fibers, and thus the "number of crossings" is no longer equivalent to "probability of intersection."

We also calculate mean fiber segment length and segment length distributions, where a segment is defined as the distance between two consecutive intersection points along a fiber, for the networks studied. In image analysis, mean coverage and mean width of fibers are readily measured, and so we specifically examine the relationship of these two key parameters to other network statistics. Thus, we set out the following four general objectives in the present paper.
(1) To develop a general methodology for characterizing nonstraight, finite-width fibers, including kinked or curly fibers.

(2) To determine the probabilistic crossing densities in a range of finite-length fiber networks, and the deterministic crossing densities in infinite systems.

(3) To determine apparent fiber densities in these networks, accounting for overlap, in order to readily allow image analysis of these systems for determination of volume fraction in thick-fiber or high-density systems.

(4) To convey the importance of the dimensions (1D vs 2D) of the fibers in determining percolation thresholds: in particular, to determine the effects of finite thickness, curliness, and density on percolation threshold in fibrous systems.

\section{CHARACTERIZATION OF CURLY, FINITE-WIDTH FIBER SYSTEMS}

\section{A. Mean curvature}

Kallmes and Corte ${ }^{1}$ introduced the concept of a curl ratio $\tau$, the ratio of fiber running length $\lambda$ to end distance $c$, to describe the degree of curliness. This quantity, however, is not sensitive to local variations in curvature. For example, a closed circular ring fiber and a closed rectangle ring fiber both have $\tau=\infty$, but have vastly different appearance, and, as we will show, properties.

The centerline of a two-dimensional (2D), curly fiber can mathematically be described via two functions, namely, $y=f(s), x=g(s), s \in[0, \lambda]$, where $f$ and $g$ are functions of the position $s$ along the direction of running length. As a substitute for curl ratio, we can calculate a "mean curvature" $\kappa$, defined as the mean value of curvature along the running length, per

$$
\begin{aligned}
\bar{\kappa} & =\frac{1}{\lambda} \int \kappa d s \\
& =\frac{1}{\lambda} \int \frac{y_{x x}}{\left(1+y_{x}^{2}\right)^{3 / 2}}\left(1+y_{x}^{2}\right)^{1 / 2} d x \\
& =\frac{1}{\lambda} \int \frac{y_{x x}}{1+y_{x}^{2}} d x
\end{aligned}
$$

and a "mean curl radius" $r$, per the usual definition as the inverse of curvature, i.e.,

$$
\bar{r}=\frac{1}{\lambda} \int \frac{1}{\kappa} d x=\frac{1}{\lambda} \int \frac{\left(1+y_{x}^{2}\right)^{2}}{y_{x x}} d x .
$$

Fibers comprising segments of circular arcs, sweeping angle $\alpha$, have mean curvature

$$
\bar{\kappa}=\frac{\alpha}{2 \sin (\alpha / 2)} .
$$

In two applications of network modeling, namely, collagens ${ }^{15}$ and carbon nanotubes, ${ }^{7,8}$ fibers present approximately as sinusoids. The running length and mean curvature of sinusoidal fibers of negligible width, defined generally by $y=A \sin (\omega x), x \in[0, d]$, are determined from 


$$
\begin{aligned}
\lambda & =\int_{0}^{\pi / \omega} \sqrt{1+A^{2} \omega^{2} \cos ^{2}(\omega x)} d x \\
& =\frac{1}{\omega} \int_{0}^{\pi} \sqrt{1+A^{2} \omega^{2} \cos ^{2}(t)} d t
\end{aligned}
$$

and

$$
\bar{\kappa}=\frac{1}{\lambda} \int_{0}^{\pi / \omega} \frac{y_{x x}}{1+y_{x}^{2}} d x=\frac{1}{\lambda} \int_{0}^{\pi} \frac{A \omega \sin t}{1+A^{2} \omega^{2} \cos ^{2} t} d t,
$$

respectively. The curl ratio for these fibers is simply

$$
\tau=\frac{\lambda}{d}=\frac{1}{\pi} \int_{0}^{\pi} \sqrt{1+A^{2} \omega^{2} \cos ^{2}(t)} d t .
$$

We can further derive an explicit relation between curl ratio $\tau$ and angular frequency $\omega$ for a sinusoid, via evaluation of the previous integral, yielding

$$
\bar{\kappa}=\frac{4}{\lambda} \tan ^{-1}(A \omega) .
$$

\section{B. Number of crossings}

Kallmes and Corte ${ }^{1}$ claimed that for curly fibers, the total number of interfiber crossings is

$$
N_{c}=\frac{\left(N_{f} \lambda\right)^{2}}{\pi S \tau^{2}}
$$

or,

$$
N_{c}=\frac{\chi^{2}}{\pi S}
$$

where $\chi$ represents the sum of the end-to-end distances for all fibers, $S$ is the area of the domain in interest. This relation is derived by assuming that the probability of intersection for two curly fibers is identical to that for two straight fibers, with the same end-to-end distances. An immediate implication of this result is that the number of crossings in a fibrous system is strongly inversely proportional to the curl ratio (i.e., $N_{c} \sim 1 / \tau^{2}$ ). Furthermore, the solution is apparently only valid within some range of $\tau$, in light of unrealistic solutions obtained for the limiting cases of straight or bent fibers, at any density. We thus reexamine calculation of the number of crossings as follows.

Statement: suppose $N_{f}$ fibers of arbitrary shapes are deposited randomly in an arbitrary two-dimensional (2D) domain of area $S$. Assuming fibers have the lengths $\lambda_{1}$, $\lambda_{2}, \ldots, \lambda_{N_{f}}$, the mathematical expectation of the crossing number is then exactly

$$
\frac{\left(\sum_{i=1}^{N_{f}} \lambda_{1}\right)^{2}-\sum_{i=1}^{N_{f}} \lambda_{i}^{2}}{\pi S} .
$$

Proof: Consider two arbitrary straight-line segments, of lengths $\xi$ and $\eta$, where $\theta$ is the angle between the two segments. The probability that these two segments intersect is the ratio of the area of a parallelogram formed by the two vectors, and the total area of the domain, namely,

$$
\frac{\xi \eta}{S}|\sin \theta|
$$

The probability of intersection of any two arbitrary segments of lengths $\xi$ and $\eta$ is then the integral of this area over $\theta \in[0, \pi]$, i.e.,

$$
p(\xi, \eta)=\frac{1}{\pi} \int_{0}^{\pi} \frac{\xi \eta}{S}|\sin \theta| d \theta=\frac{2 \xi \eta}{\pi S} .
$$

A curly fiber can always be approximated by straight segments of infinitesimal size. Thus, the number of crossing points intersection between two arbitrary fibers $\lambda_{1}$ and $\lambda_{2}$ can be determined from a summation of the probabilities of the intersections of all of their constituent straight segments, per

$$
\begin{aligned}
N_{c}\left(\lambda_{1}, \lambda_{2}\right) & =\sum_{k=1}^{m_{1}} \sum_{q=1}^{m_{2}} P\left(\xi_{k}, \eta_{q}\right) \\
& =\sum_{k=1}^{m_{1}} \sum_{q=1}^{m_{2}} \frac{2 \xi_{k} \eta_{q}}{\pi S} \\
& =\frac{2 \lambda_{1} \lambda_{2}}{\pi S}
\end{aligned}
$$

where $\xi$ and $\eta$ now represent the constituent line segments of fibers $\lambda_{1}$ and $\lambda_{2}$, respectively, and $m_{1}$ and $m_{2}$ are the segment numbers on each of the two fibers. We readily determine the total number of crossings for $\lambda_{1}, \lambda_{1}, \ldots, \lambda_{N_{f}}$ to be

$$
N_{c}=\frac{12 \sum_{i=1}^{N_{f}} \sum_{j=1, j=1}^{N_{f}} \lambda_{i} \lambda_{j}}{\pi S}=\frac{\left(\sum_{i=1}^{N_{f}} \lambda_{i}\right)^{2}-\sum_{i=1}^{N_{f}} \lambda_{i}^{2}}{\pi S},
$$

where the factor of $1 / 2$ corrects for the fact that intersections are implicitly counted twice, once on each fiber. For a large number of fibers, $N_{f} \gg 2$, we have

$$
\left(\sum_{i=1}^{N_{f}} \lambda_{i}\right)^{2} \gg \sum_{i=1}^{N_{f}} \lambda_{i}^{2}
$$

and consequently,

$$
N_{c} \approx \frac{\left(\sum_{i=1}^{N_{f}} \lambda_{i}\right)^{2}}{\pi S}=\frac{L^{2}}{\pi S},
$$

regardless of the length distribution, where $L$ is the sum of the fiber running lengths. Note that this reduced solution takes the same form as that derived by Miles, ${ }^{3,4}$ though Miles' solution was derived in the context of straight lines.

Previously, it was shown that all dependent variables can be calculated from $N_{c} \cdot{ }^{14}$ For example, the mean segment length is

$$
l_{c}=\frac{\sum_{i=1}^{N_{f}} \lambda_{i}}{2 N_{c}}=\frac{\pi S \sum_{i=1}^{N_{f}} \lambda_{i}}{2\left[\left(\sum_{i=1}^{N_{f}} \lambda_{i}\right)^{2}-\sum_{i=1}^{N_{f}} \lambda_{i}^{2}\right]} .
$$

If the fibers have the same length $\lambda$, then $N_{c}$ can be simplified as

$$
N_{c}=\frac{\lambda^{2} N_{f}\left(N_{f}-1\right)}{\pi S},
$$




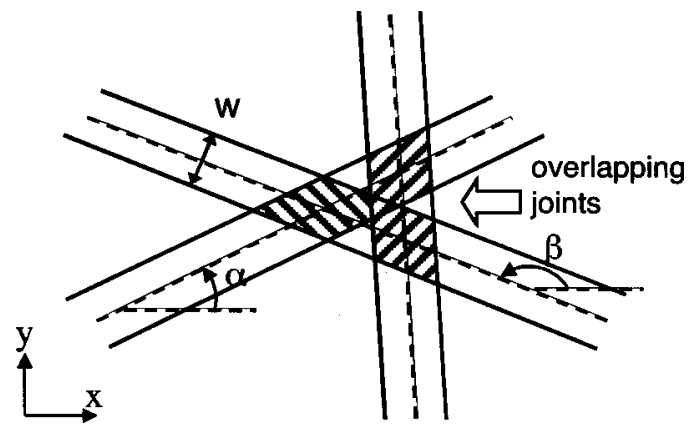

FIG. 2. A cluster formed by the overlapping junctions between three thick fibers.

where $N_{f}$ is the total number of fibers.

If we normalize the parameters, i.e., we choose the fiber length to be 1 and the domain area also to be 1 , we obtain

$$
N_{c}=\frac{N_{f}\left(N_{f}-1\right)}{\pi},
$$

and the mean segment length is then

$$
l_{c}=\frac{\pi}{2\left(N_{f}-1\right)} .
$$

\section{Effect of finite fiber width}

The preceding derivations were based on the assumption that the fiber widths are negligible. We can develop similar results, however, for systems of fibers of finite widths, from first principles. Assuming that any Poissonian system has coverage $H$ in domain $S$, deposition of a small object of area $d C$ will result in a small increment of coverage $d H$. It follows that $d H=(1-H) d C / S$. Integration of this relation immediately leads to

$$
H=1-\exp (-C / S),
$$

where $C$ is the total area of the individual objects. This general expression gives the mean coverage (or area fraction) of any homogeneous Poissonian system. ${ }^{16}$ It follows for a system of curly fibers that

$$
H=1-\exp \left(-\sum_{i=1}^{N_{f}} \frac{\lambda_{i} w_{i}}{S}\right) .
$$

\section{Image analysis and network statistics: Mean coverage and mean width}

In image analysis of porous fibrous materials, e.g., carbon nanotube sheets, ${ }^{7}$ mean coverage of the fiber phase and mean fiber width are readily obtained. These two parameters can be used to determine the number of crossings and other statistical properties of the network. Specifically, from Eqs. (17) and (20), we find that

$$
N_{c}=\frac{S \log ^{2}(1-H)}{\pi w^{2}},
$$

where $w$ represents the mean fiber width. Equation (22) clearly does not account for possible overlap between finite areas of interconnections among crossing fibers, examples of which are shown in Fig. 2. Indeed, analytical determination of the number of fibers in a particular joint would require a very tedious categorization of the connecting graphs. Thus, we reserve discussion of these properties for Sec. IV, in which we describe results of Monte Carlo simulations of such systems.

The mean segment length $l_{c}$ (measured equivalently as either the mean centerpoint-to-centerpoint length, or the mean length of the sides of the internal polygons) is invariant to fiber width, as pointed out earlier by Miles. ${ }^{3}$ Combination of Eqs. (16) and (21) gives an explicit relation for this parameter, namely,

$$
l_{c}=\frac{-\pi w}{2 \log (1-H)} .
$$

\section{E. Probability of intersection}

For systems containing fibers of very high curvature, the crossing events are not independent of one another. Otherwise, locations of crossing points in a fibrous system are Poissonian, with the segment length distribution

$$
f(l)=\rho \exp (-\rho l)
$$

where $\rho$ is the density of crossing points, or the reciprocal of mean segment length, $1 / l_{c}$. The probability that an arbitrary fiber is isolated, is exactly the probability that no intersections occur on that fiber, namely,

$$
P_{\text {iso }} \approx \exp (-\rho \lambda) .
$$

For two arbitrary fibers of lengths $\lambda_{1}$ and $\lambda_{2}$, the probability of intersection is

$$
P\left(\lambda_{1}, \lambda_{2}\right)=\frac{2 \lambda_{1} \lambda_{2}}{\pi S},
$$

assuming the fibers intersect at a single point. This solution is not valid in circumstances where fibers can intersect at multiple points. For example, for two identical, circular fibers, the probability of intersection is

$$
P\left(\lambda_{1}, \lambda_{2}\right)=\frac{4 \pi r^{2}}{S}=\frac{\lambda_{1} \lambda_{2}}{\pi S},
$$

which is exactly half of that derived in Eq. (26).

Kallmes and Corte ${ }^{1}$ derived an expression for the mean common area between two fibers as

$$
\delta_{c}=\frac{\pi}{2} w^{2} .
$$

This result was based on Mack's earlier derivation ${ }^{17}$ of the probability of intersection between two arbitrary, twodimensional, convex domains of areas $\delta_{1}, \delta_{2}$, and perimeters $U_{1}$ and $U_{2}$, respectively. The solution,

$$
P_{\mathrm{int}}=\frac{\delta_{1}+\delta_{2}+U_{1} U_{2} /(2 \pi)}{S},
$$

is invalid for curly fibers, because they are not convex. For example, for two circular fibers having circumferential lengths $\lambda_{1}$ and $\lambda_{2}$, the probability of intersection is equal to 


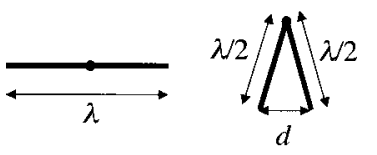

(a)

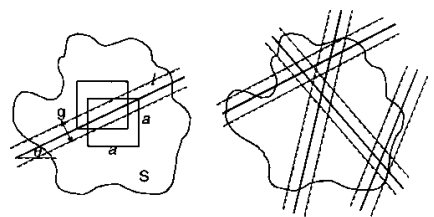

(a) (b)
$N_{c} / 2$, with $N_{c}$ given by Eq. (12), since there are exactly two crossing points for each intersection. As a result

$$
P_{\text {int }}=\frac{N_{c}}{2}=\frac{\lambda_{1} \lambda_{2}}{\pi S}
$$

whereas Eq. (29) gives

$$
P_{\text {int }}=\frac{0+0+\left(2 \lambda_{1}\right)\left(2 \lambda_{2}\right) /(2 \pi)}{S}=\frac{2 \lambda_{1} \lambda_{2}}{\pi S} .
$$

The inconsistency between the solutions of Eqs. (30) and (31) illustrates the dependence of probability of intersection on fiber waviness. Specific comparisons are detailed in Sec. IV.

\section{F. Percolation threshold}

Percolation is a collective event arising from intersections among individual members in a network. Since fiber waviness affects intersection probability, it also affects the network percolation threshold. From earlier work on straight fibers, ${ }^{18,19}$ we know that, for fibers of length $\lambda$ and critical density $\rho_{c}$ (number of fibers per unit area) at percolation, $\rho_{c} \lambda^{2} \approx 5.59 \pm 0.05$ is an invariant.

For a network formed by curly fibers of uniform shape, we can show that $\gamma$, given by

$$
\gamma=\rho \lambda^{2}
$$

is also invariant with respect to different length scales, at percolation onset. The parameter $\gamma$ can therefore be used conveniently as a standard measurement of "percolation threshold." We support this with a brief explanation. Considering an arbitrary fibrous network, which percolates at $\rho_{c}$, we can enlarge any subdomain arbitrarily, without altering the fibers' shapes and relative positions, or percolation status. Supposing we increase each edge dimension by factor $m$, i.e., the domain area is magnified by $m^{2}$, fibers' running length becomes $m \lambda$ and fiber density $\rho$ is reduced to $\rho_{c} / m^{2}$. Hence, $\gamma=\rho_{c} / m^{2 *}(m \lambda)^{2}=\rho_{c} \lambda^{2}$. On the other hand, if constituent fibers form closed domains, an alternative quantity, "reduced density" $\eta=\rho_{c} A$, may be used to serve as a measurement of percolation threshold, where $A$ represents the area formed by a closed fiber ring.

We consider a specific case of the effect of curl ratio $\tau$ on $\gamma$. Suppose a straight fiber is bent about its mid point as shown in Fig. 3. For an end-to-end distance of zero, the system properties match those of a system having straight fibers of length $\lambda / 2$. Thus, at the percolation point we have

$$
\rho_{c}(\lambda / 2)^{2} \approx 5.59 \text {. }
$$

Per the previous definition, we have

$$
\gamma_{c}=\rho_{c} \lambda^{2}=5.59 \times 4=22.36 .
$$

FIG. 4. Schematic showing the notation used in derivation of percolation properties for infinite fibers.

Comparing this property to that obtained for the systems of a circular fiber "rings," the percolation density would be equivalent to that of fully overlapping disks, i.e.,

$$
\rho_{c} \pi r^{2}=67.634 \%
$$

based on Quintanilla's recent measurement. ${ }^{20}$ This leads to

$$
\gamma_{c}=\rho_{c}(2 \pi r)^{2} \approx 8.499 \text {. }
$$

Therefore, $\gamma_{c}$ is clearly a function of curl ratio $\tau$, i.e.,

$$
\gamma_{c}-\psi(\tau)
$$

\section{CHARACTERIZATION OF STRAIGHT, INFINITE FIBER SYSTEMS}

Consider a fibrous network constructed by homogeneous deposition of infinite straight lines of density $\rho$, that is, the total length of line segments is $\rho$ per unit area. If a simulation window of finite size is used to measure the statistical properties of the network, scaling effects will be inevitably involved. The percolation problem of such a finite zone is nontrivial, since the network in question does not always percolate. Nevertheless, if we define the term "percolation" as the condition in which a single line, and not a cluster of line segments, crosses opposite sides of a domain, it is possible to derive some fundamental solutions from the statistical theory, without resorting to estimation from Monte Carlo simulation.

\section{A. Percolation probability}

The first problem is to determine the probability that no lines span the boundaries of a given domain. Consider the intersection of a line and a square domain. Clearly, there is a band region in which the line at a fixed angle spans opposite sides of the domain, as shown in Fig. 4(a). The width $g$ of the band region is

$$
g=a|| \cos \theta|-| \sin \theta||,
$$

where $\theta \in[0, \pi]$ represents the inclination angle of the line, and $a$ is the length of a side of the square. If a single line is deposited into a large domain of area $S$, the probability of finding an arbitrary square region percolated by the line is

$$
p=\frac{g l}{S}=\frac{a l|| \cos \theta|-| \sin \theta||}{S},
$$

where $\theta \in[0, \pi / 4]$. If there are multiple random lines with length $l_{i}(i=1,2, \ldots)$, as shown in Fig. 4(b), we have 


$$
\begin{aligned}
\sum a l_{j}|| \cos \theta_{i}|-| \sin \theta_{i}|| & =\frac{4}{\pi} a L \int_{0}^{\pi / 4}|\cos \theta-\sin \theta| d \theta \\
& =\frac{4(\sqrt{2}-1)}{\pi} a L
\end{aligned}
$$

where $L$ represents the total length of the line segments. The union area of all the line segments is then given by

$$
S\left[1-\exp \left(-\frac{4(\sqrt{2}-1)}{\pi} a \frac{L}{S}\right)\right] .
$$

The probability of finding that the square is percolated by at least one line is equivalent to the ratio of the union area and the domain area, namely,

$$
p=\frac{S_{\text {union }}}{S}=1-\exp \left(-\frac{4(\sqrt{2}-1)}{\pi} a \rho\right) .
$$

When the size of the square is sufficiently small, i.e., $a \rho$ $\ll 1$, this probability becomes after Taylor series expansion

$$
p \approx 0.527 a \rho \text {. }
$$

Likewise, the probability of finding that all of the lines percolate the square domain is

$$
1-\exp \left\{\left(\frac{4(\sqrt{2}-1)}{\pi}-1\right) a \rho\right\} .
$$

Trivially, the probability of finding no line segments residing inside the square is

$$
\exp \left(-a \rho \frac{4}{\pi} \sqrt{2} \int_{0}^{\pi / 4} \cos \theta d \theta\right)=\exp \left(-\frac{4}{\pi} a \rho\right) .
$$

This general method also applies to other domain shapes. For example, the probability of finding that no lines are contained inside an equilateral triangular domain of side length $a$ is

$$
\exp \left(-a \rho \frac{6}{\pi} \int_{0}^{\pi / 6} \cos \theta d \theta\right)=\exp \left(-\frac{3 a \rho}{\pi}\right)
$$

In more general, for an $n$-side equilateral polygon of side length $a$, when $n$ is an even integer, the probability of finding no lines across the polygon would be

$$
\begin{gathered}
\exp \left(-a \rho \frac{1}{\sin (\pi / n)} \frac{2 n}{(n-2) \pi} \int_{0}^{(n-2) \pi / 2} \cos \theta d \theta\right) \\
=\exp \left(-a \rho \frac{2 n}{(n-2) \pi} \cos \frac{\pi}{n}\right)
\end{gathered}
$$

and when $n$ is an odd integer, the probability becomes

$$
\begin{gathered}
\exp \left(-a \rho \frac{\cos \frac{\pi}{2 n} 2 n}{\sin \frac{\pi}{n}(n-2) \pi} \int_{0}^{(n-2) \pi / 2} \cos \theta d \theta\right) \\
=\exp \left(-a \rho \frac{2 n}{(n-2) \pi} \cos \frac{\pi}{n} \cos \frac{\pi}{2 n}\right)
\end{gathered}
$$

For a circular domain of diameter $a$, the probability of finding no lines crossing the domain is simply

$$
\exp (-a \rho)
$$

\section{B. Mean length of line segments}

Supposing an infinite line at inclination angle $\theta$ crosses an $a \times a$ square domain, the mean length of the line segments truncated by the four sides of the square can be written as

$$
\begin{aligned}
\lambda & =a \frac{1}{2}[(1+\tan \theta)+(1-\tan \theta)] \sec \theta /(1+\tan \theta) \\
& =\frac{a}{\cos \theta+\sin \theta},
\end{aligned}
$$

where $\theta \in[0, \pi / 4]$. Therefore, the mean length of the truncated lines at all angles is

$$
\lambda=\frac{4}{\pi} \int_{0}^{\pi / 4} \frac{a}{\cos \theta+\sin \theta} d \theta=\frac{a}{\sqrt{2}} \ln (\sqrt{2}+1) \approx 0.6232 a .
$$

Applying Eq. (15) yields the relation between the number of intersections and the total number of fibers,

$$
N_{c}=\frac{\left(0.6232 a N_{f}\right)^{2}}{\pi a^{2}} \approx 0.1236 N_{f}^{2} .
$$

Thus, the number of crossings is solely a function of the number of fibers, for straight fibers.

\section{SIMULATIONS: CURLY AND STRAIGHT FIBER SYSTEMS}

We verified several of our solutions for finite fibers using Monte Carlo simulations. First, we investigated use of our analytical solution for the number of crossings in systems of curly fibers, with specific emphasis on determination of verification of the independence of the number of crossings on fiber shape.

Next, we investigated the effect of finite width on mean coverage and mean width numerically, since these parameters are critically important in image analysis of fibrous systems. Finally, we performed simulations of probability of intersection, as a precursor to determination of percolation threshold in various curly, kinked, and straight-fiber systems.

\section{A. Number of crossings}

To verify our solution, Eq. (9), we studied curly fibers of sinusoidal shape of the form $y=A \sin (\omega x)$. To fiber lengths controllable, periodic boundaries were applied to the networks. Meanwhile, all parameters, including $\lambda, A$, and $\omega$, are assumed fixed. In all simulations, $A=0.05$ and unit running lengths for fibers were used (Fig. 5). Simulations were repeated for different number of fibers; results are shown in Fig. 6. We can see that excellent agreement is achieved between the numerical results and the theoretical solution using Eq. (9). Clearly, the number of fiber crossings is invariant to fiber shape. 


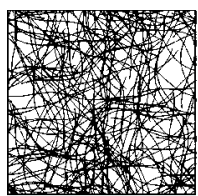

(a)

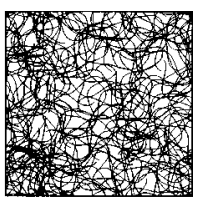

(b)
FIG. 5. Random networks of 100 fibers of unit running length and amplitude $A=0.05$ for (a) $\omega=10$ and (b) $\omega=20$.

\section{B. Finite fiber widths: Image analysis and network statistics}

Our previous analytic solution for the number of crossings in a fibrous system, Eq. (9), is valid only for very thin fibers. Because of the tedious nature of an analytic calculation of the number of crossings in a finite-width network, we conducted Monte Carlo simulations to develop empirical relations for a range of such systems. The joint formed by two crossing fibers of width $w$, and inclinations $\alpha$ and $\beta$, comprises a rhombus, of side length

$$
\mu=\frac{w}{\sin (\alpha-\beta)} .
$$

The four vertices are

$$
\begin{aligned}
& x-x_{0}=\frac{ \pm \cos \alpha \pm \cos \beta}{\sin (\alpha-\beta)} w \\
& y-y_{0}=\frac{ \pm \sin \alpha \pm \sin \beta}{\sin (\alpha-\beta)} w
\end{aligned},
$$

where $x_{0}$ and $y_{0}$ represent the $x$ and $y$ positions of the crossing point of the two center lines (or, fiber axes). Once the four vertices of each joint rhombus are located, the connectivity between joints can be examined by determining the intersection status between each individual boundary line of each rhomboidal joint. The total number of clusters decreases with increasing fiber width, since joints inevitably merge, forming fewer, larger clusters. This intuitive result was verified by simulation results shown in Fig. 7, wherein

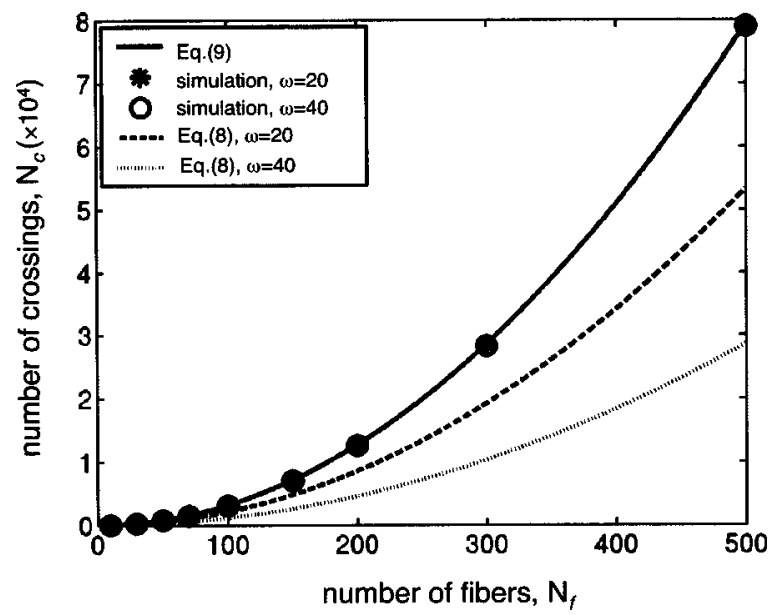

FIG. 6. Simulations, and present and prior analytical results for the number of fiber crossings vs number of fibers, for systems of varying fiber waviness.

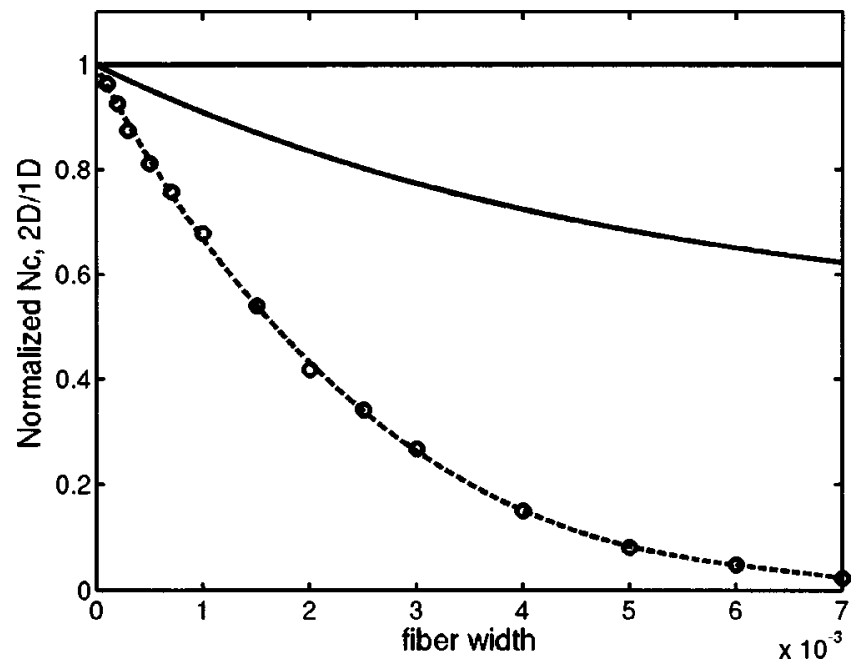

FIG. 7. Number of fiber crossings as a function of number of fibers. Overlapping junctions are considered to comprise single crossings. Results are normalized by the number of crossings, without consideration of fiber thickness.

our Monte Carle results are compared to an analytic result reported earlier by Corte, ${ }^{14}$ namely,

$$
N_{c}=\frac{1}{2}\left(1+e^{-N_{f} \lambda w}\right) N_{f}^{2} \lambda^{2} / \pi S .
$$

Our simulations were performed in a unit cell, using 200 fibers of unit length. Fiber widths were varied to determine the effect of width on $N_{c}$. Clear differences are seen between simulations performed here and the prior analytical solution. ${ }^{14}$

\section{Probability of intersection}

To illustrate the dependence of intersection probability on fiber waviness for a specific shape of fiber, a series of simulations were performed using two sinusoidal fibers of half period. A unit size simulation window and two fibers of unit length were used in these simulations. Ten thousand simulations were performed in each case and the results were averaged. In the results presented in Fig. 8, the intersection probability was normalized against the result at $\tau=\infty$, and interpreted as a function of the curl ratio $\tau$, using Eq. (6). Because the two fibers act as straight fibers of lengths $\lambda$ and $\lambda / 2$ in the two extreme situations of zero and infinite curl ratios, respectively, we have

$$
p_{\text {int }}(\tau=0)=\frac{2 \lambda^{2}}{\pi S}
$$

and

$$
p_{\text {int }}(\tau=\infty)=\frac{2(\lambda / 2)^{2}}{\pi S}=\frac{\lambda^{2}}{2 \pi S} .
$$

Thus, the normalized probability monotonically decreases with curl ratio, asymptotically approaching a value of 0.25 as $\tau \rightarrow \infty$. 


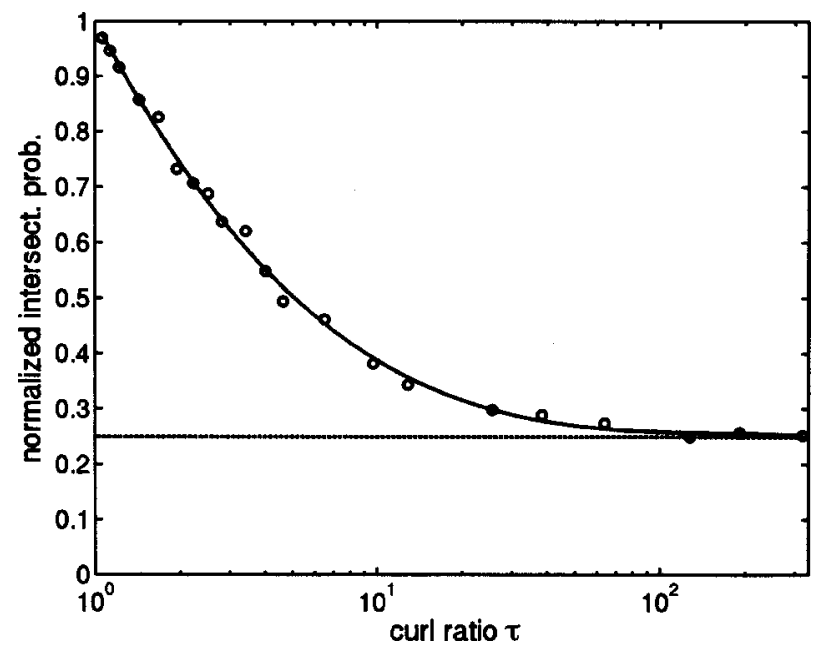

FIG. 8. Intersection probability as a function of curl ratio, between two sinusoidal fibers of length one-half period. Results are normalized by probability of intersection between two straight fibers.

\section{Percolation threshold: curly, kinked, and straight fiber systems}

Dependene of percolation threshold on curl ratio was investigated for 2D, curly fibers having three distinct shapes as shown in Fig. 9(a): (1) sinusoid, (2) triangle, and (3) rectangle. These representative geometries could provide insight as to the effect of the type of waviness on percolation properties in general. For each of the three types, we constructed a square simulation window and deposited fibers randomly onto this domain. Monte Carlo simulations were performed to determine the $\rho$ at which the geometric percolation across a pair of opposite boundaries arose. Each fiber was divided into 20 , straight-line segments so that the interfiber connectivity would be detected via checking the connectivity between line segment pairs. Fifty simulations were performed at each density and the results were averaged. Periodic boundaries were applied. Fiber number was held to 30005000 in each simulation so that the fiber length was less than $1 / 10$ of the window size in order to minimize the scaling effect. For each shape, we held fiber length constant for various curl ratios, as shown in Fig. 9(b). The results are presented in Figs. 10(a) and 10(b). These results were also tabulated in Table 1 using linear interpolation at evenly spaced values of $\mu(=1 / \tau)$. Simulation errors were estimated to approximately $\Delta \rho= \pm 0.08$.

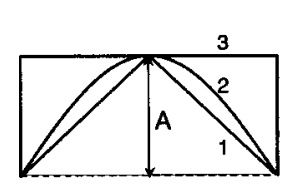

(a)

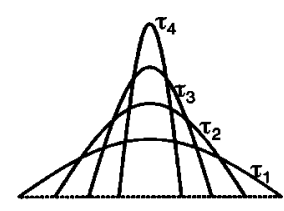

(b)
FIG. 9. Fiber shapes investigated for percolation simulations, including (a) three distinct types of curves, and (b) sinusoidal curves having various curl ratios.

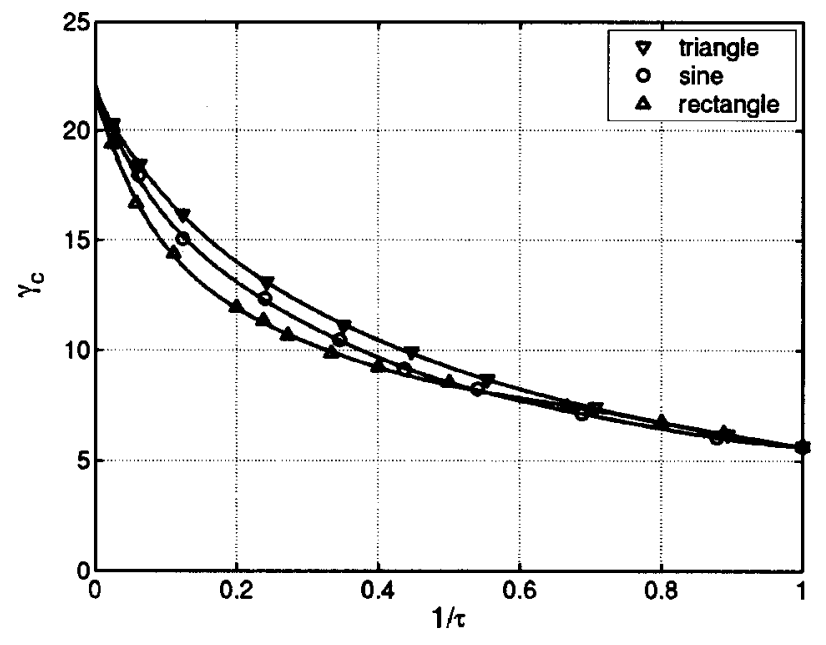

(a)

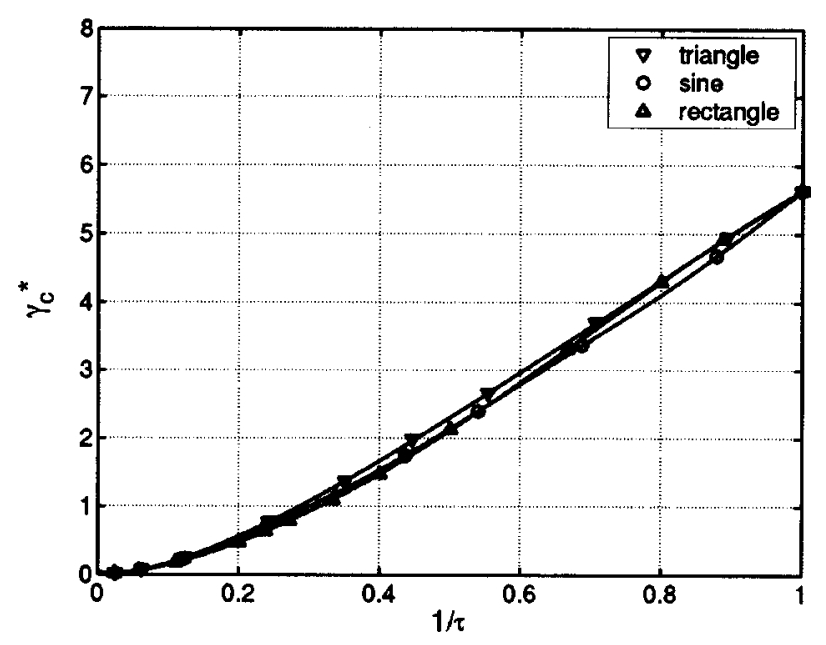

(b)

FIG. 10. (a) Percolation threshold $\gamma_{c}$ as a function of curl ratio, where $\gamma_{c}$ is expressed as $N_{f} \lambda^{2}$, and $\lambda$ is fiber length. (b) Percolation threshold $\gamma_{c}^{*}$ as a function of curl ratio, where $\gamma_{c}^{*}$ is expressed as $N_{f} c^{2}$ instead of $N_{f} \lambda^{2}$, and $c$ is the end-to-end distance.

\section{DISCUSSION/CONCLUSIONS}

\section{A. Number of crossings in straight- and curly-fiber systems}

Clearly, Eq. (8), an expression for number of crossings in systems of curly fibers derived by Kallmes and Corte, ${ }^{1}$ is in error, as shown both by our own analytic solution of Eq. (9), and comparison of both solutions with Monte Carlo simulations shown in Fig. 6. In fact, Eq. (8) is an acceptable estimate only when $\tau$ is very close to 1 . The immediate implication of our new solution is that the number of crossings is independent of fiber waviness.

We additionally found that the number of crossings is solely a function of the number of fibers in infinite fiber systems. This result is useful in practice, because our solution, Eq. (52), can be used readily to deduce the statistical 
TABLE I. Comparison of percolation thresholds among three different shapes of fibers.

\begin{tabular}{lccc}
\hline \hline $1 / \tau$ & $\gamma_{c}($ sinusoidal $)$ & $\gamma_{c}($ triangle $)$ & $\gamma_{c}$ (rectangle) \\
\hline 1.00 & 5.63 & 5.63 & 5.63 \\
0.95 & 5.78 & 5.89 & 5.87 \\
0.90 & 5.97 & 6.16 & 6.13 \\
0.85 & 6.20 & 6.44 & 6.42 \\
0.80 & 6.46 & 6.75 & 6.73 \\
0.75 & 6.76 & 7.08 & 7.04 \\
0.70 & 7.06 & 7.44 & 7.32 \\
0.65 & 7.39 & 7.84 & 7.59 \\
0.60 & 7.73 & 8.26 & 7.85 \\
0.55 & 8.12 & 8.73 & 8.12 \\
0.50 & 8.56 & 9.26 & 8.43 \\
0.45 & 9.08 & 9.83 & 8.80 \\
0.40 & 9.69 & 10.48 & 9.24 \\
0.35 & 10.39 & 11.20 & 9.76 \\
0.30 & 11.18 & 12.02 & 10.36 \\
0.25 & 12.08 & 12.95 & 11.06 \\
0.20 & 13.12 & 14.04 & 11.92 \\
0.15 & 14.40 & 15.35 & 13.06 \\
0.10 & 16.08 & 16.97 & 14.74 \\
0.05 & 18.47 & 19.04 & 17.39 \\
0.00 & 22.51 & 22.51 & 22.51 \\
\hline \hline
\end{tabular}

properties of fibers from the result of image analyses, and thus enables the proper selection of modeling parameters thereafter.

In finite-width fiber systems, we observed significant discrepancies among our simulation results and Corte's ${ }^{14}$ prior analytical formula. This prior result, Eq. (55), is applicable only for systems having volume fractions below $10 \%$.

\section{B. Probability of intersection, and percolation thresholds}

We found that even slight waviness can have a nonnegligible effect on the probability of intersection in fibrous systems, as shown by comparison of simulations of intersections in wavy and straight-fiber systems, and comparisons with classical solutions for straight fibers (see Fig. 8). For example, for a curl ratio of $\tau=1.1$, use of Eq. (26) to calculate $P$ results in an overestimation of $4 \%$; for $\tau=1.2$, the resulting overestimation is $8 \%$. When $\tau=1.5$ (roughly equivalent to that of a half circle), the overestimation exceeds $20 \%$.

The dependence of probability of intersection on fiber waviness led us to numerically investigate a dependence of percolation on fiber waviness. Three representative geometries for fibers, namely, sinusoidal, triangular, and rectangular configurations, were used to systematically examine percolation thresholds. In the simulations, unit simulation windows were used and fiber lengths between 0.05 and 0.1 were selected, in order to minimize scale effect. The number of fibers in each realization was varied from 2000 to 5000, depending on curl ratio. One hundred simulations were performed in each case, and results were averaged. Several important conclusions can be drawn from these percolation simulations.

(1) Waviness significantly affects the geometric percolation threshold in fibrous networks. Increases in curl ratio $\tau$ leads to increase in fiber density required for percolation. This observation logically follows our findings that increases in curl ratio reduces probability of intersection within arbitrary pairs of fibers. For example, for sinusoidal fibers of slight waviness $1 / \tau=0.9, \gamma_{c}$ is found to be 5.97 , an increase of $6 \%$ over that for straight fibers; if $1 / \tau=0.7, \gamma_{c}$ is 7.06 , with an increase of $25 \%$ compared to that of straight fibers. If $1 / \tau=0.5, \gamma_{c}$ is 8.56, an increase of $52 \%$.

(2) Percolation threshold is a monotonic function of curl ratio, with values between 5.63 and 22.51 , for each of the three shapes of fibers investigated. This is again consistent with our previous finding that probability of intersection is a monotonic function of curl ratio.

(3) Percolation threshold does not differ much for various shapes of fibers having identical curl ratio. For example, sinusoidal fiber systems studied had percolation thresholds very close to those of the other two shapes studied, with variations of less than $10 \%$ for all values of $\tau$. Therefore, use of a sinusoidal model appears reasonable in many practical applications wherein the percolation threshold is sought.

In summary, we found that the number of intersections in a Poissonian fibrous network is independent of fiber waviness. However, waviness in the fiber shape raises geometric percolation thresholds. In a network comprised of infinite straight fibers, the number of crossings depends only on the number of fibers. Intersection probability between individual fibers, as well as the clustering of junctions in thick-fiber systems, were also studied in detail. Future work will include application of these findings to image analysis of curly-fiber systems, including collagens.

\section{ACKNOWLEDGMENTS}

Support for this work provided by the W. M. Keck Foundation, and from DARPA and ONR through the Synthetic Multifunctional Materials (SMFM) Program (Dr. Leo Christodoulou and Dr. Steve Fishman, Program Monitors) is gratefully acknowledged. Support from an NSF PECASE (Sastry) is also gratefully acknowledged.

${ }^{1}$ O. Kallmes and H. Corte, Tappi J. 43, 737 (1960).

${ }^{2}$ S. Goudsmit, Rev. Mod. Phys. 17, 321 (1945).

${ }^{3}$ R. E. Miles, Proc. Natl. Acad. Sci. U.S.A. 52, 901 (1964a).

${ }^{4}$ R. E. Miles, Proc. Natl. Acad. Sci. U.S.A. 52, 1157 (1964b).

${ }^{5}$ H. Wang, B. E. Layton, and A. M. Sastry, Diabetes-Metab. Res. 19, 288 (2003).

${ }^{6}$ B. E. Layton, A. M. Sastry, H. Wang, K. A. Sullivan, E. L. Feldman, T. E. Komoroski, and M. A. Philbert, J. Biomech. (in press).

${ }^{7}$ L. Berhan, A. M. Sastry, E. Munoz, M. Selvidge, and R. Baughman, J. Appl. Phys. 95, 4335 (2004).

${ }^{8}$ L. Berhan, Y. B. Yi, and A. M. Sastry, J. Appl. Phys. 95, 5027 (2004).

${ }^{9}$ C. W. Wang, L. Berhan, and A. M. Sastry, ASME J. Eng. Mater. Technol. 122, 450 (2000).

${ }^{10}$ A. M. Sastry, C. W. Wang, and L. Berhan, Probab. Methods Fatigue Fract. 200, 229 (2001).

${ }^{11}$ L. Berhan and A. M. Sastry, J. Compos. Mater. 37, 715 (2003).

${ }^{12}$ P. I. Richards, Proc. Natl. Acad. Sci. U.S.A. 52, 1160 (1964). 
${ }^{13}$ J. C. Tanner, J. Appl. Probab. 20, 778 (1983).

${ }^{14}$ H. Corte, Statistical Geometry of Random Fibre Networks, Structure, Solid Mechanics and Engineering Design, Proceedings of the Southampton 1969 Civil Engineering Materials Conference, Part I, edited by M.

Te'eni (Wiley-Interscience, New York, 1969), p. 341.

${ }^{15}$ B. E. Layton and A. M. Sastry, ASME J. Biomech. Eng. (in press).
${ }^{16}$ G. P. Wadsworth and J. G. Bryan, Introduction to Probability and Random Variables (McGraw-Hill, New York, 1960).

${ }^{17}$ C. Mack, Proc. Cambridge Philos. Soc. 50, 581 (1954).

${ }^{18}$ I. Balberg and N. Binenbaum, Phys. Rev. B 28, 3799 (1983).

${ }^{19}$ J. Asikainen and T. Ala-Nissila, Phys. Rev. E 61, 5002 (2000).

${ }^{20}$ J. Quintanilla, S. Torquato, and R. M. Ziff, J. Phys. A 33, 399 (2000). 